\title{
Diagnosis of bacterial vaginosis by a new multiplex peptide nucleic acid fluorescence in situ hybridization method
}

António Machado, Joana Castro, Tatiana Cereija, Carina Almeida, Nuno Cerca

Bacterial vaginosis (BV) is one of most common vaginal infections. However, its diagnosis by classical methods reveals low specificity. Our goal was to evaluate the accuracy diagnosis of 150 vaginal samples with research gold standard methods and our Peptide Nucleic Acid (PNA) probes by Fluorescence in situ Hybridization (FISH) methodology. Also, we described the first PNA-FISH methodology forBV diagnosis, providing results in approximately 3 hours. The results showed a sensitivity of $84.6 \%$ (95\% confidence interval (Cl), from 64.3 to $95.0 \%)$ and a specificity of $97.6 \%(95 \% \mathrm{Cl}$, from 92.6 to $99.4 \%)$, demonstrating the higher specificity of the PNA-FISH method and showing false positive results in BV diagnosis commonly obtained by the classical methods. This methodology combines the specificity of PNA probes for Lactobacillus species and G. vaginalis visualization and the calculation of the microscopic field by Nugent score, allowing a trustful evaluation of the bacteria present in vaginal microflora and avoiding the occurrence of misleading diagnostics. Therefore, the PNA-FISH methodology represents a valuable alternative for $\mathrm{BV}$ diagnosis. 


\section{António Machado; Joana Castro; Tatiana Cereija; Carina Almeida; Nuno Cerca*}

3 Centre of Biological Engineering, LIBRO - Laboratory of Research in Biofilms Rosário Oliveira,

4 University of Minho, Campus de Gualtar, 4710-057, Braga, Portugal.

5 *Corresponding author: nunocerca@ceb.uminho.pt

6 Tel.: (+351) 253604400

7 Fax: (+351) 253604429

8

9 
12 Bacterial vaginosis (BV) often exhibits high prevalence, high relapse rates and associated 13 complications, which renders this infection of global importance (Falagas, Betsi \& Athanasiou, 2007;

14 Tibaldi et al., 2009). BV is associated with increased taxonomic richness and diversity (Oakley et al., 15 2008) and is normally characterized by a decrease in vaginal lactobacilli and a simultaneous increase in 16 the anaerobes population (Tibaldi et al., 2009). Therefore, vaginal bacterial communities differ 17 dramatically between healthy patients and patients with BV, where G. vaginalis is present in over $90 \%$ 18 of BV cases (Verstraelen \& Swidsinski, 2013). G. vaginalis role is still controversial since this 19 bacterium is also present in $10-40 \%$ of healthy women (Aroutcheva et al., 2001; Hickey \& Forney, 20 2014; Silva et al., 2014); however, recent evidence suggests that the presence of G. vaginalis biofilms, 21 instead of dispersed cells, are in fact an indication of BV (Verstraelen \& Swidsinski, 2013). 22 Furthermore, we recently demonstrated that G. vaginalis has significant higher virulence potential than 23 other $29 \mathrm{BV}$ associated species, including higher cytotoxicity and biofilm formation ability (Alves et 24 al., 2014). We also provided evidence that G. vaginalis biofilms can establish synergistic relationships 25 with other BV anaerobes (Machado, Jefferson \& Cerca, 2013), further highlighting its pivotal role on 26 BV development.

The most frequently used method for BV diagnosis is the physician's assessment by Amsel 29 clinical criteria (Forsum, Hallén \& Larsson, 2005). This method is fairly subjective and is based on the 30 observation of the following symptoms: vaginal fluid with $\mathrm{pH}$ above 4.5; positive "whiff test" 31 (detection of fishy odor upon $10 \%$ potassium hydrogen addition); presence of clue cells (vaginal 32 epithelial cells covered by bacteria) on microscopic examination of vaginal fluid; and homogeneous 33 milky vaginal discharge. At least three of the four symptoms described above must be present to 
34 establish a positive BV diagnosis (Amsel et al., 1983). Despite the fact that the Amsel criteria does not 35 require intensive training, it is not the most appropriate method to diagnose $\mathrm{BV}$, due to its low specificity (Dickey, Nailor \& Sobel, 2009).

Alternatively, laboratory diagnosis is based on the Nugent score analysis, a microscopic method that quantifies three different bacteria morphotypes presented in the vaginal smears (Nugent, Krohn \& Hillier, 1991). These authors have created a Gram stain scoring system based on the evaluation of the following morphotypes: large gram-positive rods (Lactobacillus spp. morphotypes); small gramvariable rods (G. vaginalis morphotypes); small gram-negative rods (Bacteroides spp. morphotypes); and curved gram-variable rods (Mobiluncus spp. morphotypes). Each morphotype is quantified from 0 to 4 with regard to the number of morphotypes observed in the microscopic fields of the Gram-stained vaginal smear. The vaginal microflora is then classified in normal microflora (scores of 0 to 3 ) or as BV (scores of 7 to 10), based on the sum of each morphotype score (Livengood, 2009; Nugent, Krohn \& Hillier, 1991). However, the evaluation of smears is also subjective and user dependent (Sha et al., 2005). Furthermore, due to its low specificity, the Nugent method also considers intermediate microflora whenever the final score is between 4 and 6.

Although both methodologies are easy and fast to perform, they do not provide a robust diagnosis of BV. When combined, these standard tests have a sensitivity and specificity of 81 and $70 \%$ (Forsum et al., 2005), respectively. To improve BV diagnosis, several new molecular methodologies have been proposed with fluorescence in situ hybridization (FISH) a very promising alternative. This technique combines the simplicity of microscopic observation and the specificity of DNA/rRNA hybridization, allowing the detection of selected bacterial species and morphologic visualization (Justé,

5 Thomma, \& Lievens, 2008; Nath, 2000). Peptide Nucleic Acid (PNA) probes are used instead of natural nucleic acids to improve FISH efficiency because they enable quicker and more specific 


.

hybridization (Lefmann et al., 2006; Peleg et al., 2009; Wilson et al., 2005). These types of probes, in which bases are linked by a neutral peptide backbone, avoid the repulsion between the negatively charged phosphate backbone characteristics of DNA/DNA hybridization (Stender et al., 2002). Since PNA is a synthetic molecule, probes are also resistant against cytoplasmic enzymes, such as nucleases and proteases (Amann \& Fuchs, 2008). In addition, the hybridization step can be performed efficiently under low salt concentrations, which promotes the destabilization of rRNA secondary structures and consequently improves the access to target sequences (Almeida et al., 2009; Cerqueira et al., 2008). All these advantages have made PNA-FISH a new promising tool for diagnosis and therapy-directing techniques, providing already a rapid and accurate diagnosis of several microbial infections (Hartmann et al., 2005; Shepard et al., 2008; Søgaard et al., 2007; Trnovsky et al., 2008).

We have previously developed a multiplex PNA-FISH method, able to specifically quantify in vitro Lactobacilli spp. and G. vaginalis adhered to HeLa cells (Machado et al., 2013). To determine the feasibility of our novel PNA-FISH method as a diagnostic tool for BV, we have blind tested our multiplex methodology on vaginal samples from Portuguese women and compared those results with the laboratory microscopic derived method using the Nugent score.

\section{Material \& Methods}

\section{Vaginal sample collection and preparation}

A total of 200 vaginal fluid samples were obtained, after informed consent, as approved by the Institutional Review Board (Subcomissão de Ética para as Ciências da Vida e Saúde) of University of Minho (process SECVS 003/2013). The vaginal samples were collected for Gram staining and FISH 
79 procedures, using the culture swab transport system (VWR, CE0344, Italy). The extraction procedure

80 from transport media was elaborated in accordance with Money's guidelines to avoid misleading in the

81 Nugent score analysis of the vaginal swabs (Money, 2005). These swabs were brushed against the

82 lateral vaginal wall to collect the vaginal fluid sample, placed into the culture swab transport media and

83 immediately stored at $4{ }^{\circ} \mathrm{C}$. First, the vaginal samples were used for Gram stain procedure, as described

84 by Nugent and colleagues (Nugent et al., 1991). Next, swabs were immersed in $1 \mathrm{ml}$ of phosphate

85 buffer saline (PBS) and the remaining vaginal material collected by centrifugation at 17,000 $g$ during 5

$86 \mathrm{~min}$ at room temperature. Afterwards, the pellet was resuspended in $2 \mathrm{ml}$ of saline solution $(0.9 \% \mathrm{NaCl})$

87 and finally diluted 1:10 in saline solution or PBS to eliminate possible contaminants that could 88 interfere with FISH procedures, as previously described (Machado et al., 2013).

\section{Classification of vaginal smears according to Nugent score}

Vaginal samples evaluation was performed using the Nugent criteria score (Nugent et al., 1991). Briefly, Gram stained vaginal smears were examined under oil immersion objective (1000x magnification) and 10-15 microscopic fields were evaluated for each sample. The composite score was

94 grouped into three categories, scores $0-3$ being normal, $4-6$ being intermediate, and 7-10 being definite

95 bacterial vaginosis. Finally, the smears that showed scores between $0-3$ and 7-10 were selected for 96 further study, as normal (-) and BV (+) samples, respectively. Meanwhile, the smears with a Nugent 97 score of 4-6 or with incomplete epidemiological data were rejected from our study.

\section{Fluorescent in situ hybridization}


101 test. For each sample, $20 \mu \mathrm{L}$ of the final suspension were spread on glass slides. The slides were air-

102 dried prior to fixation. Next, the smears were immersed in $4 \%$ (wt/vol) paraformaldehyde (Fisher 103 Scientific, United Kingdom) followed by 50\% (vol/vol) ethanol (Fisher Scientific, United Kingdom)

104 for $10 \mathrm{~min}$ at room temperature on each solution. After the fixation step, the samples were covered 105 with $20 \mu \mathrm{L}$ of hybridization solution containing $10 \%$ (wt/vol) dextran sulphate (Fisher Scientific, 106 United Kingdom), $10 \mathrm{mM} \mathrm{NaCl}$ (Sigma, Germany), 30\% (vol/vol) formamide (Fisher Scientific, 107 United Kingdom), 0.1\% (wt/vol) sodium pyrophosphate (Fisher Scientific, United Kingdom), 0.2\% 108 (wt/vol) polyvinylpyrrolidone (Sigma, Germany), 0.2\% (wt/vol) ficoll (Sigma, Germany), $5 \mathrm{mM}$ 109 disodium EDTA (Sigma, Germany), 0.1\% (vol/vol) triton X-100 (Sigma, Germany), 50 mM Tris-HCl 110 (at pH 7.5; Sigma, Germany) and $200 \mathrm{nM}$ of each PNA probe (Lactobacillus spp. PNA Probe: Lac663 111 probe, Alexa Fluor 488-ACATGGAGTTCCACT; HPLC purified > 90\%; Gardnerella vaginalis PNA 112 Probe: Gard162 probe, Alexa Fluor 594-CAGCATTACCACCCG; HPLC purified > 90\%). 113 Subsequently, the smears were covered with coverslips and incubated in moist chambers at the 114 hybridization temperature $\left(60^{\circ} \mathrm{C}\right)$ during $90 \mathrm{~min}$. Next, the coverslips were removed and a washing 115 step was performed by immersing the slides in a pre-warmed washing solution for 30 min at the same 116 temperature of the hybridization step. This solution consisted of $5 \mathrm{mM}$ Tris base (Fisher Scientific, 117 United Kingdom), $15 \mathrm{mM} \mathrm{NaCl}$ (Sigma, Germany) and 0.1\% (vol/vol) triton X-100 (at pH 10; Sigma, 118 Germany). Finally, the glass slides were allowed to air dry. 
Prior to microscopy, one drop of non-fluorescent immersion oil (Merck, Germany) was added

122 to either slides and covered with coverslips. Microscopic visualization was performed using an

123 Olympus BX51 (Olympus Portugal SA, Portugal) epifluorescence microscope equipped with a CCD

124 camera (DP72; Olympus, Japan) and filters capable of detecting the two PNA probes (BP 470-490,

125 FT500, LP 516 sensitive to the Alexa Fluor 488 molecule attached to the Lac663 probe and BP 530-

126550 , FT 570, LP 591 sensitive to the Alexa Fluor 594 molecule attached to the Gard162 probe).

In each experimental assay, a negative control was performed simultaneously, in which all the

128 steps described above were carried out, but where no probe was added in the hybridization step.

129 Finally, 20 random regions of each glass slide were photographed. All images were acquired using

130 Olympus CellB software using a total magnification of $\& 1000$.

\section{Statistical analysis}

133 The data was analyzed to calculate sensitivity, specificity, accuracy, positive and negative

134 likelihood ratios (PLR and NLR, respectively) of the PNA-FISH methodology, with 95\% confidence 135 intervals (CI), using a clinical online statistical software (www.vassarstats.net/clin1.html; accessed 136 2014) (Senthilkumar, 2006). The classic Nugent criteria score was used as the diagnostic true.

137

\section{Results and Discussion}

On this prospective study, 150 vaginal samples were used to compare BV diagnosis by the 140 classic Nugent criteria and our PNA-FISH methodology. As shown in Table 1, the main characteristics 141 of the sample population used to validate our method, mirrors what has been described in other main 
142 epidemiological studies, namely (1) the overall rate of positive BV cases (17\%) in the general 143 population (Koumans et al., 2007; Li et al., 2014; Jespers et al., 2014), (2) an association between 144 previous BV infections and BV positive diagnostic (Bilardi et al., 2013; Guedou et al., 2013), (3) a 145 higher risk factors for women using the pill, instead of a condom (Bradshaw et al., 2013; Guedou et al., 146 2013), and (4) the history of previous pregnancy higher in women with BV (Africa, Nel \& Stemmet, 147 2014; Mengistie et al., 2014).

As shown in Table 2, PNA-FISH method was able to diagnose 121 from a total of 124 healthy 149 cases and capable to categorize 22 positive cases from a total of $26 \mathrm{BV}$ cases, when compared with the 150 standard Nugent score. PNA-FISH methodology was capable to illustrate clear differences between 151 healthy and BV samples, showing specific detection of Lactobacillus spp. and G. vaginalis species 152 directly in clinical samples. In fact, a typically healthy sample and a BV sample exhibited a totally 153 different vaginal microflora, such as UM300 and UM235 samples, respectively, being clue cells and $G$. 154 vaginalis augmentation easily detected in UM235 sample (see Fig. 1). However, some discrepancies 155 were also found between the two methodologies, more exactly in 7 vaginal samples. In fact, 4 vaginal 156 samples were positive for BV by Gram staining but negative by PNA-FISH evaluation while the others 1573 vaginal samples were negative for BV by Gram staining but positive by PNA-FISH evaluation. It is 158 well known that conventional BV diagnosis accuracy is highly dependent on the training and 159 experience of the technician due to the unspecific staining of the Gram method (Simoes et al., 2006), 160 which might explain some of the discrepant results observed.

161 To better evaluate the diagnostic value of the proposed PNA-FISH approach, the technique 162 performance was assessed by determining the following parameters: specificity, sensitivity, accuracy, 163 PLR, NLR. Based on these results, an experimental specificity of $97.6 \%$ (95\% CI, 92.6 to 99.4\%) 164 and sensitivity of $84.6 \%$ (95\% CI, 64.3 to $95.0 \%)$ were obtained for the BV diagnosis by our PNA- 
165 FISH method (Table 2). Therefore, a high accuracy was also obtained for our PNA-FISH method, 166 more exactly, a value of $95.3 \%$ (95\% CI, 89.2 to $98.3 \%)$.

167 Regarding the likelihood ratios the PNA-FISH method evidenced a PLR of 34.97 and a NLR of 0.16.

168 So, the specificity and the NLR values show the test ability to correctly identify as normal person that 169 do not have BV. While, the low NLR obtained, in fact, tells that the probability of having BV is much 170 decreased (0.16) for a negative PNA-FISH result. Moreover, our experimental specificity revealed to 171 be superior than Nugent's Gram stain system specificity (83\%) (Schwebke et al., 1996). Therefore, our 172 method was able to correctly identify $97.6 \%$ of those patients previously classified with normal vaginal 173 flora making PNA-FISH a trustful method to ensure a healthy diagnosis and avoiding false positive 174 results.

175 In opposition, the sensitivity and PLR values demonstrated a strong association between a positive 176 result for BV diagnostic and the probability of the patient having indeed BV. In this case, the high PLR 177 tells us how increased is the probability of having BV $(35 \times)$, if the test result is positive. The 178 sensitivity value was in fact lower than expected, taking in consideration our previous in vitro 179 experiments, where we have reached to a sensitivity of $100 \%$ (95\% CI, from 81.5 to $100.0 \%)$ 180 (Machado et al., 2013). Despite the experimental sensitivity (84.6\%) was slight lower than the 181 specificity of the Gram staining by Nugent score (89\%) (Schwebke et al., 1996), it was nevertheless 182 higher than the Amsel criteria sensitivity $(60 \%)$ determined by Gallo and colleagues (Gallo et al., 183 2011). It is important to refer that other bacterial species, with similar Gram staining morphology, 184 could be at high number in the samples leading to an incorrect classification of BV according to 185 Nugent criteria. In fact, Verhelst and colleagues presented evidences that infers a lack of accuracy in 186 the interpretation of the results in Gram stain by Nugent score in their clinical results (Verhelst et al., 187 2005). Forsum and colleagues also found discrepancies in scoring bacterial cell types, when 188 pleomorphic lactobacilli and other kinds of bacteria could be regarded as G. vaginalis cells, leading to 
189 an incorrect BV diagnosis (Forsum et al., 2002; Schwiertz et al., 2006). Also, it is important to refer

190 that G. vaginalis may vary in size and form, from round to more elongated, where there is no defined

191 border to separate them from the lactobacilli morphotypes (Forsum et al., 2002), thus illustrating again

192 problems in the accuracy of the smears interpretation. These facts suggest that the sensitivity value is

193 likely to be underestimated.

194 Overall, despite the cost effective nature of the Nugent score, PNA-FISH appears to be an 195 accurate method for detecting BV from vaginal samples, maintaining similar complexity as the 196 previous standard method.

198 Conclusions

In conclusion, in this study we described the first PNA-FISH methodology applied for BV 200 diagnosis, and the parameters evaluated have proved it potential as a diagnostic tool. The performance 201 characteristics of this PNA-FISH method also suggest that it might be a reliable alternative to the 202 Amsel criteria and Gram stain under Nugent score. Despite our sample size was somewhat small, the 203 population at study was representative from what has been described by many other epidemiological 204 studies, therefore validating this prospective study.

\section{References}

208 Africa CW, Nel J, Stemmet M. 2014. Anaerobes and bacterial vaginosis in pregnancy: virulence 209 factors contributing to vaginal colonisation. International Journal of Environmental Research and 210 Public Health 11:6979-7000. doi: 10.3390/ijerph110706979. 
212 Almeida C, Azevedo NF, Iversen C, Fanning S, Keevil CW, Vieira MJ. 2009. Development and 213 application of a novel peptide nucleic acid probe for the specific detection of Cronobacter 214 genomospecies (Enterobacter sakazakii) in powdered infant formula. Applied and Environmental 215 Microbiology 75: 2925-2930. doi:10.1128/AEM.02470-08.

217 Alves P, Castro J, Sousa C, Cereija TB, Cerca N. 2014. Gardnerella vaginalis outcompetes 29 218 other bacterial species isolated from patients with bacterial vaginosis, using in an in vitro biofilm 219 formation model. Journal of Infectious Diseases 210: 593-596.

221 Amann R, Fuchs BM. 2008. Single-cell identification in microbial communities by improved 222 fluorescence in situ hybridization techniques. Nature Reviews Microbiology 6: 339-348. 223 doi:10.1038/nrmicro1888

Amsel R, Totten PA, Spiegel CA, Chen KC, Eschen-bach DHK. 1983. Nonspecific vaginitis. 226 Diagnostic criteria and microbial and epidemiologic associations. American Journal of Medicine 74: $227 \quad 14-22$.

Aroutcheva AA, Simoes JA, Behbakht K, Faro S. 2001. Gardnerella vaginalis isolated from 230 patients with bacterial vaginosis and from patients with healthy vaginal ecosystems. Clinical Infectious 231 Diseases 33: 1022-1027. 
235 emotional, sexual and social impact of living with recurrent bacterial vaginosis. PloS one 8: e 74378. 236 doi: 10.1371/journal.pone.0074378.

Bradshaw CS, Vodstrcil LA, Hocking JS, Law M, Pirotta M, Garland SM, De Guingand D, 239 Morton AN, Fairley CK. 2013. Recurrence of bacterial vaginosis is significantly associated with 240 posttreatment sexual activities and hormonal contraceptive use. Clinical infectious diseases: an official publication of the Infectious Diseases Society of America 56: 777-786. doi: 10.1093/cid/cis1030.

Cerqueira L, Azevedo NF, Almeida C, Jardim T, Keevil CW, Vieira MJ. 2008. DNA mimics for

the rapid identification of microorganisms by fluorescence in situ hybridization (FISH). International Journal of Molecular Sciences 9: 1944-1960. doi:10.3390/ijms9101944

Dickey LJ, Nailor MD, Sobel JD. 2009. Guidelines for the treatment of bacterial vaginosis : focus on tinidazole. Therapeutics and Clinical Risk Management 5: 485-489.

Falagas ME, Betsi GI, Athanasiou S. 2007. Probiotics for the treatment of women with bacterial vaginosis. Clinical Microbiology Infection 13: 657-664.

Forsum U, Hallén A, Larsson P. 2005. Bacterial vaginosis-a laboratory and clinical diagnostics enigma. Acta Pathologica, Microbiologica et Immunologica Scandinavica 113: 153-161. 
259 criteria of bacterial vaginosis. Acta Pathologica, Microbiologica et Immunologica Scandinavica 110: $260 \quad 811-818$.

262 Gallo MF, Jamieson DJ, Cu-Uvin S, Rompalo A, Klein RS, Sobel JD. 2011. Accuracy of clinical 263 diagnosis of bacterial vaginosis by human immunodeficiency virus infection status. Sexually 264 Transmitted Diseases 38: 270-274. doi:10.1097/OLQ.0b013e3181fce4eb $10.1186 / 1471-2334-13-208$.

Hartmann H, Stender H, Schäfer A, Autenrieth IB, Kempf VA. 2005. Rapid Identification of 272 Staphylococcus aureus in Blood Cultures by a Combination of Fluorescence In Situ Hybridization 273 Using Peptide Nucleic Acid Probes and Flow Cytometry. Journal of Clinical Microbiology 43: 4855274 4857. doi:10.1128/JCM.43.9.4855

276 Hickey RJ, Forney LJ. 2014. Gardnerella vaginalis does not always cause bacterial vaginosis. 277 Journal of Infectious Diseases 210: 1682-1683. 
281 bacterial vaginosis in different sub-populations of women in sub-Saharan Africa: a cross-sectional 282 study. PloS one 9: e109670. doi: 10.1371/journal.pone.0109670.

284 Justé A, Thomma BP, Lievens B. 2008. Recent advances in molecular techniques to study microbial 285 communities in food-associated matrices and processes. Food Microbiology 25: 745-761. 286 doi:10.1016/j.fm.2008.04.009

The prevalence of bacterial vaginosis in the United States, 2001-2004; associations with symptoms, 290 sexual behaviors, and reproductive health. Sexually Transmitted Diseases 34: 864-869.

\section{Lefmann M, Schweickert B, Buchholz P, Göbel UB, Ulrichs T, Seiler P, Theegarten D, Moter A.}

2006. Evaluation of peptide nucleic acid-fluorescence in situ hybridization for identification of

295 Microbiology 44: 3760-3767. doi:10.1128/JCM.01435-06

Li, XD, Wang CC, Zhang XJ, Gao GP, Tong F, Li X, Hou S, Sun L, Sun YH. 2014. Risk factors

298 for bacterial vaginosis: results from a cross-sectional study having a sample of 53,652 women.

299 European journal of clinical microbiology \& infectious diseases: official publication of the European 300 Society of Clinical Microbiology 33: 1525-1532. doi: 10.1007/s10096-014-2103-1. 
302 Livengood CH. 2009. Bacterial Vaginosis: An Overview for 2009. Reviews in Obstetrics and 303 Gynecology 2: 28-37.

305 Ma L, Lv Z, Su J, Wang J, Yan D, Wei J, Pei S. 2013. Consistent condom use increases the 306 colonization of Lactobacillus crispatus in the vagina. PLoS One 8:e70716. doi: 307 10.1371/journal.pone.0070716.

308

309 Machado A, Almeida C, Salgueiro D, Henriques A, Vaneechoutte M, Haesebrouck F, Vieira MJ, 310 Rodrigues L, Azevedo NF, Cerca N. 2013. Fluorescence In Situ Hybridization Method Using Peptide 311 Nucleic Acid Probes for rapid detection of Lactobacillus and Gardnerella spp. BMC microbiology 312 13:82.

314 Machado A, Jefferson KK, Cerca N. 2013. Interactions between Lactobacillus crispatus and 315 Bacterial Vaginosis (BV)-Associated Bacterial Species in Initial Attachment and Biofilm Formation. 316 International Journal of Molecular Sciences 14:12004-12.

318 Menard JP, Fenollar F, Henry M, Bretelle F, Raoult D. 2008. Molecular quantification of 319 Gardnerella vaginalis and Atopobium vaginae loads to predict bacterial vaginosis. Clinical Infectious 320 Diseases 47: 33-43. doi:10.1086/588661

Mengistie Z, Woldeamanuel Y, Asrat D, Adera A. 2014. Prevalence of bacterial vaginosis among 323 pregnant women attending antenatal care in Tikur Anbessa University Hospital, Addis Ababa, 324 Ethiopia. BMC Research Notes 7:822. doi: 10.1186/1756-0500-7-822. 
326 Money D. 2005. The laboratory diagnosis of bacterial vaginosis. Canadian Journal of Infectious 327 Diseases \& Medical Microbiology 16:77-9. doi:10.1086/18159532.

Nath JK. 2000. A review of fluorescence in situ hybridization (FISH): current status and future 330 prospects. Biotechnic \& Histochemistry 75: 54-78.

Nugent R, Krohn M, Hillier S. 1991. Reliability of Diagnosing Bacterial Vaginosis Is Improved by a Standardized Method of Gram Stain Interpretation. Journal of Clinical Microbiology 29: 297-301.

Oakley BB, Fiedler TL, Marrazzo JM, Fredricks DN. 2008. Diversity of human vaginal bacterial 336 communities and associations with clinically defined bacterial vaginosis. Applied and Environmental Microbiology 74: 4898-909. doi:10.1128/AEM.02884-07

GM. 2009. Utility of peptide nucleic acid fluorescence in situ hybridization for rapid detection of 341 Acinetobacter spp. and Pseudomonas aeruginosa. Journal of Clinical Microbiology 47: 830-832. doi:10.1128/JCM.01724-08

Schwebke JR, Hillier SL, Sobel JD, McGregor JA. 1996. Validity of the vaginal gram stain for the diagnosis of bacterial vaginosis. Obstetrics \& Gynecology 88: 573-576.

Schwiertz A, Taras D, Rusch K, Rusch V. 2006. Throwing the dice for the diagnosis of vaginal complaints? Annals of Clinical Microbiology and Antimicrobials 5: 1-7. doi:10.1186/1476-0711-5-4 
350 Senthilkumar MP. 2006. Statistics on the web. Annals of The Royal College of Surgeons of England 351 88: 325-332. doi:10.1308/003588406X106469

353 Sha BE, Chen HY, Wang QJ, Zariffard MR, Cohen MH, Spear GT. 2005. Utility of Amsel 354 criteria, Nugent score, and quantitative PCR for Gardnerella vaginalis, Mycoplasma hominis, and 355 Lactobacillus spp. for diagnosis of bacterial vaginosis in human immunodeficiency virus-infected 356 women. Journal of Clinical Microbiology 43:4607-12.

358 Shepard JR, Addison RM, Alexander BD, Della-Latta P, Gherna M, Haase G, Hall G, Johnson 359 JK, Merz WG, Peltroche-Llacsahuanga H, Stender H, Venezia RA, Wilson D, Procop GW, Wu 360 F, Fiandaca MJ. 2008. Multicenter evaluation of the Candida albicans/Candida glabrata peptide 361 nucleic acid fluorescent in situ hybridization method for simultaneous dual-color identification of $C$. 362 albicans and C. glabrata directly from blood culture bottles. Journal of Clinical Microbiology 46: 50363 55. doi:10.1128/JCM.01385-07 Silva D, Henriques A, Cereija T, Martinez-de-Oliveira J, Miranda M, Cerca N. 2014. Prevalence 366 of Gardnerella vaginalis and Atopobium vaginae in Portuguese women and association with risk 367 factors for bacterial vaginosis. International Journal of Gynecology \& Obstetrics 124:178-9.

369 Simoes JA, Discacciati MG, Brolazo EM, Portugal PM, Dini DV, Dantas MC. 2006. Clinical 370 diagnosis of bacterial vaginosis. International Journal of Gynecology \& Obstetrics 94: 28-32. 371 doi:10.1016/j.ijgo.2006.04.013 
373 Søgaard M, Hansen DS, Fiandaca MJ, Stender H, Schønheyder HC. 2007. Peptide nucleic acid

374 fluorescence in situ hybridization for rapid detection of Klebsiella pneumoniae from positive blood 375 cultures. Journal of Medical Microbiology 56: 914-917. doi:10.1099/jmm.0.46829-0

376

Stender H, Fiandaca M, Hyldig-Nielsen JJ, Coull J. 2002. PNA for rapid microbiology. Journal of Microbiological Methods 48: 1-17. doi:10.1016/S0167-7012(01)00340-2

380 Tibaldi C, Cappello N, Latino MA, Masuelli G, Marini S, Benedetto C. 2009. Vaginal and 381 endocervical microorganisms in symptomatic and asymptomatic non-pregnant females : risk factors 382 and rates of occurrence. Clinical Microbiology and Infection 15: 670-679.

Trnovsky J, Merz W, Della-Latta P, Wu F, Arendrup MC, Stender H. 2008. Rapid and accurate identification of Candida albicans isolates by use of PNA FISH Flow. Journal of Clinical Microbiology 46: 1537-1540. doi:10.1128/JCM.00030-08

Temmerman M, Vaneechoutte M. 2005. Comparison between Gram stain and culture for the characterization of vaginal microflora : Definition of a distinct grade that resembles grade I microflora and revised categorization of grade I microflora. BMC Microbiology 5: 61. doi:10.1186/1471-2180-561

Verstraelen H, Swidsinski A. 2013. The biofilm in bacterial vaginosis: implications for epidemiology, 395 diagnosis and treatment. Current Opinion in Infectious Diseases 26: 86-89. 
397 Wilson DA, Joyce MJ, Hall LS, Reller LB, Roberts GD, Hall GS, Alexander BD, Procop GW.

398 2005. Multicenter Evaluation of a Candida albicans Peptide Nucleic Acid Fluorescent In Situ

399 Hybridization Probe for Characterization of Yeast Isolates from Blood Cultures. Journal of Clinical

400 Microbiology 43: 2909-2912. doi:10.1128/JCM.43.6.2909

401

402 Witt A, Petricevic L, Kaufmann U, Gregor H, Kiss H. 2002. DNA hybridization test: rapid 403 diagnostic tool for excluding bacterial vaginosis in pregnant women with symptoms suggestive of 404 infection. Journal of Clinical Microbiology 40: 3057-3059. 


\section{1}

Fluorescence microscopy pictures of Lactobacillus spp., Gardnerella vaginalis and others bacteria species from a healthy (UM300) and BV (UM235) vaginal clinical samples.

Fluorescence microscopy pictures of Lactobacillus spp., Gardnerella vaginalis and others bacteria species from a healthy (UM300) and BV (UM235) vaginal clinical samples by specific PNA probes (Lac663 and Gard162) associated with Alexa Fluor 488 and 594 fluorochromes and DAPI staining, respectively. (a) green filter; (b) red filter; (c) blue filter; (d) overlay of the three previous filters. As shown in the green filter (a), UM300 (healthy) and UM235 (BV) samples showed the presence of Lactobacillus spp. species but only BV sample demonstrated an elevated G. vaginalis concentration in the vaginal swabs (red filter (b)), which they proved to stablish clue cells by overground the vaginal epithelial cells in the blue filter (c). Therefore, both vaginal swab samples exhibited a totally different vaginal microflora, as finally we may observed in the overlay of the filters (d), being clue cells and $G$. vaginalis augmentation easily detected in UM235 sample.

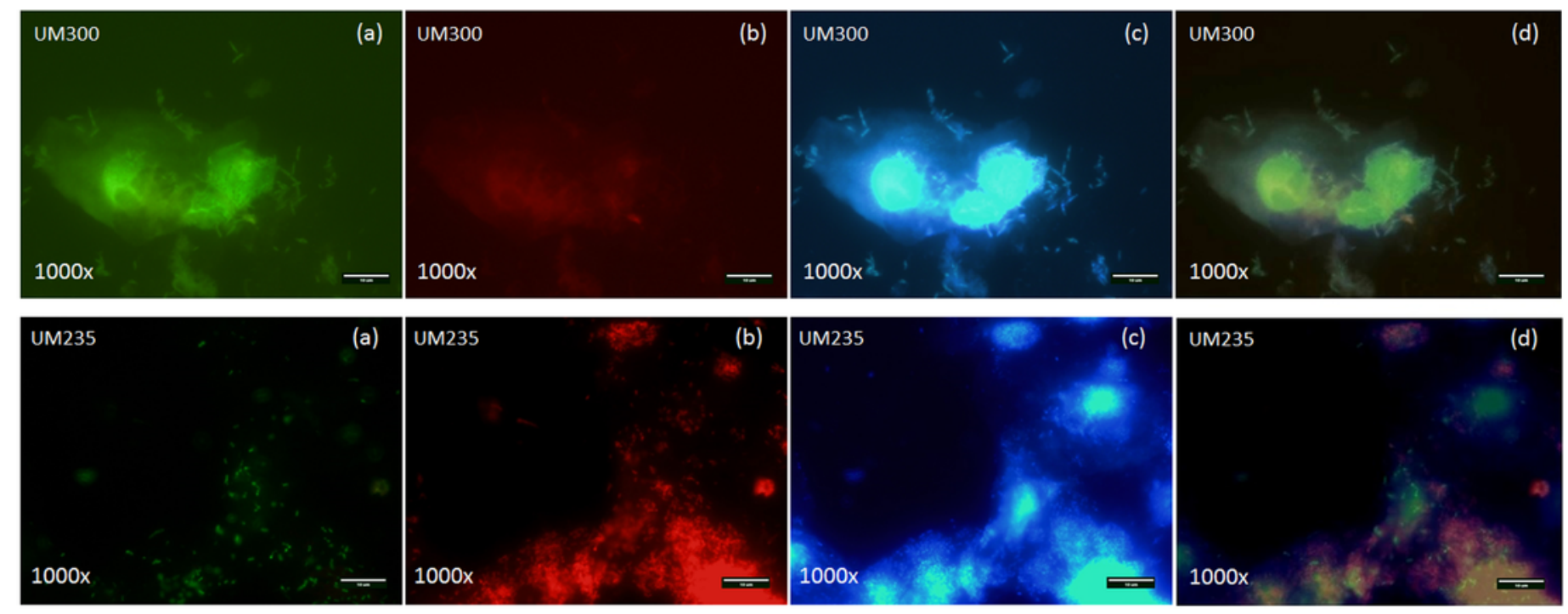




\section{Table $\mathbf{1}$ (on next page)}

Characteristics of the population of study ( $n=150$ )

Characteristics of the population of study $(n=150)$. The samples classification as normal or BV was performed according the Nugent score. 
2 Table 1. Characteristics of the population of study $(\mathrm{n}=150)$. The samples classification as normal or 3 BV was performed according the Nugent score.

\begin{tabular}{lcc}
\multicolumn{1}{c}{ Variables } & Women with normal & Women with BV \\
& flora $(\mathbf{n = 1 2 4})$ & (n=26) \\
\hline Age (years) & $30.2 \pm 11.42$ & $32.5 \pm 9.7$ \\
With children (\%) & & \\
No & 68.5 & 50.0 \\
Yes & 27.4 & 50.0 \\
Pregnant women (\%) & 4.0 & 0.0 \\
Previously diagnosed with bacterial vaginosis (\%) & 16.9 & 38.5 \\
Contraception (\%) & & \\
No contraception & & 15.4 \\
Pill & 8.9 & 61.5 \\
Condom & 54.0 & 11.5 \\
Other & 25.8 & 15.4 \\
\hline
\end{tabular}

Data are mean \pm standard deviation or $\mathrm{n}(\%)$.

5 
Table 2 (on next page)

Comparison between PNA-FISH method versus Gram staining using Nugent score criteria for BV diagnosis. 
2 Table 2. Comparison between PNA-FISH method versus Gram staining using Nugent score criteria for

3 BV diagnosis.

\begin{tabular}{|c|c|c|c|}
\hline \multirow{2}{*}{ PNA-FISH results } & \multicolumn{3}{|c|}{ Nugent results } \\
\hline & $\mathrm{BV}+$ & $\mathrm{BV}-$ & Total \\
\hline $\mathrm{BV}+$ & 22 & 3 & 25 \\
\hline BV - & 4 & 121 & 125 \\
\hline Total & 26 & 124 & 150 \\
\hline \multicolumn{4}{|c|}{ Statistical analysis of PNA-FISH method } \\
\hline & $\begin{array}{l}\text { Estimated } \\
\text { value }\end{array}$ & $\begin{array}{c}\text { Lower } \\
\text { limit }\end{array}$ & $\begin{array}{c}\text { Upper } \\
\text { limit }\end{array}$ \\
\hline Sensitivity & $84.6 \%$ & $64.3 \%$ & $95.0 \%$ \\
\hline Specificity & $97.6 \%$ & $92.6 \%$ & $99.4 \%$ \\
\hline Accuracy & $95.3 \%$ & $89.2 \%$ & $98.3 \%$ \\
\hline Positive likelihood & 34.97 & 11.30 & 108.24 \\
\hline Negative likelihood & 0.16 & 0.06 & 0.39 \\
\hline
\end{tabular}

